\title{
CoSenS: a Collecting and Sending Burst Scheme for Performance Improvement of IEEE 802.15.4
}

\author{
Bilel Nefzi \\ LORIA - Nancy University INPL \\ Campus Scientifique - BP 239 - 54506 \\ Vandoeuvre-Les-Nancy Cedex, France \\ Email: Bilel.Nefzi@loria.fr
}

\author{
Ye-Qiong Song \\ LORIA - Nancy University INPL \\ Campus Scientifique - BP 239 - 54506 \\ Vandoeuvre-Les-Nancy Cedex, France \\ Email: Ye-Qiong.Song@loria.fr
}

\begin{abstract}
IEEE 802.15.4 is considered as the de facto standard for wireless sensor networks (WSN). However, the increased probability of collision at heavy load caused by the inherent behavior of CSMA/CA degrades the performance of WSN in terms of throughput and energy consumption. This led some to propose other MAC layer solutions to tackle these problems. Most of them are TDMA-based which provides deterministic medium access. This makes them not scalable in general for large scale WSN. Contrary to that trend, we retain CSMA/CA's simplicity and good scalability properties and propose CoSenS, a collecting and sending burst scheme, that is implemented on the top of CSMA/CA. The idea of CoSenS is that a router does not retransmit packets as they arrive. Instead, it collects data from its children and other neighbor routers during a period of time that we call waiting period (WP), queue them until the expiration of that period and then sends them into a burst during a period of time that we call transmission period (TP). Simulation results show that this scheme greatly enhances throughput, end to end delay and transmission success rate (reliability). In addition, the scheme exhibits a nice property of self-adaptability of transmission periods between neighbor routers.
\end{abstract}

keywords: IEEE 802.15.4, QoS, MAC protocols, CSMA/CA, Wireless Sensor Networks, performance.

\section{INTRODUCTION}

MAC protocol design for wireless sensor networks (WSNs) has been intensively investigated by the research community [1], [2], [3], etc. We observe nowadays the convergence towards IEEE802.15.4 [4], the only MAC largely accepted and based on which products are commercially available.

IEEE802.15.4 MAC performs well in a light traffic environment, but the performance quickly degrades in heavy traffic case because of the inherent behavior of CSMA/CA (collisions) on which IEEE802.15.4 MAC is based. This is unacceptable for most of WSN applications. For example, when a fire is detected in the forest, a burst of messages will be generated thus stressing the high throughput functionality of the MAC protocol. Therefore, the MAC layer in WSN must be capable of achieving low delay, high throughput and reliability in these situations.

Some improvements exist. For industrial applications (and real-time ones), TDMA scheme over IEEE802.15.4 is the most popular solution (e.g., WirelessHART [7]). GTS mechanism of IEEE 802.15.4 and its improvement (Koubaa et al. [6]) are also possible solutions. However, the use of TDMA scheme needs careful network configuration for efficient time-slot allocation and it is not scalable in general for large scale WSN and not robust for automatically adapting to the changes in the network (nodes mobility and death, environment changes, etc).

So one of our research challenges is to find much simpler solution which should only use COTS IEEE 802.15.4 compliant chips, does not require any configuration effort and can self adapt to the dynamic network evolutions.

In this paper, based on the simple idea that CSMA/CA can be improved using burst transmission mode (IEEE 802.15.4 does support this mode), a very simple Collecting and Sending burst Scheme, CoSenS, has been proposed. Using this scheme, the performance of the network is greatly improved in terms of delay, throughput and reliability. In addition, the fact to queue the packets at routers allows us to design efficient scheduling algorithms for providing differentiated QoS and packet aggregation mechanisms.

Related Work: to our best knowledge, no work has been done in the MAC layer to improve the intrinsic performance of CSMA/CA protocol in WSN. Most of the proposed MAC protocols, including T-MAC [2], B-MAC [1], etc, focused on improving energy consumption at the cost of throughput and end-to-end delay degradation. In fact, they are all based on either the unslotted CSMA/CA protocol of IEEE 802.15.4 or the IEEE 802.11 [11] version (although, we observe nowadays a convergence toward IEEE 802.15.4). Our design can be used conjointly with these protocols or extended to support the techniques used therein (mainly duty-cycling mechanisms) to improve energy. A first extension of this framework has been already done to tackle energy and throughput tradeoff [12].

The rest of the paper is organized as follows. CoSenS design is detailed next. The performance study by simulations is given in Sec. III. Section IV concludes the paper.

\section{CoSEnS DESIGN}

\section{A. General description}

We consider a 2-tiers architecture network composed of routers to which are associated simple nodes (typically FFD and RFD of ZigBee). Routers form an ad-hoc network. In this case, any routing protocol, like AODV or "Hierarchical Tree Routing" (HTR) of ZigBee for instance, can be used 


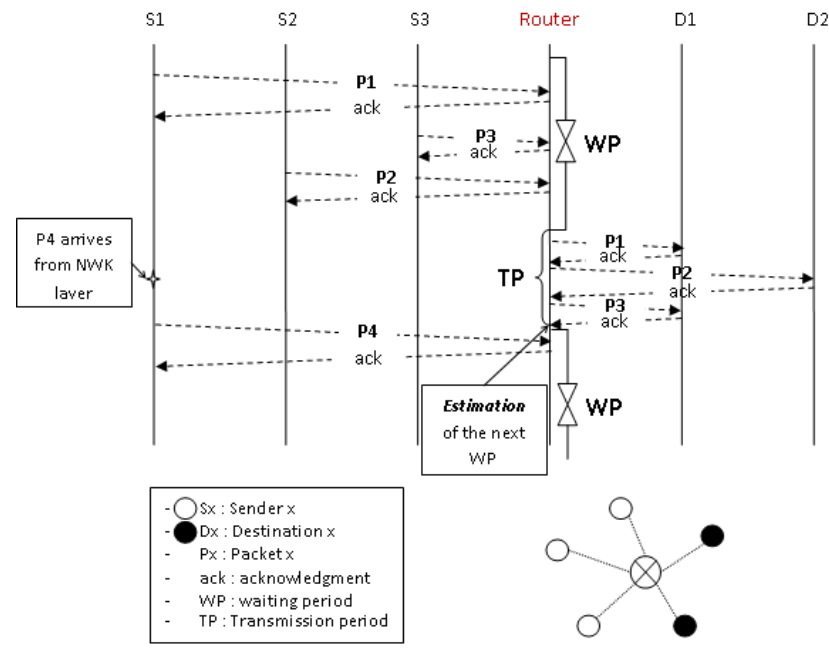

Fig. 1. An example of how CoSenS works.

to establish routes between routers. The network formation protocol is out of the scope of this article.

The basic medium access protocol for all nodes (simple nodes and routers) is CSMA/CA. We used the unslotted version proposed by IEEE 802.15.4 standard which is widely implemented in sensor chips. CoSenS is implemented on the top of CSMA/CA protocol. It is enabled for routers and disabled for simple nodes by default (in the rest of the paper, routers are used to describe CoSenS operating mode). The MAC layer operates on the top of the "2450 MHz DSSS IEEE 805.15.4" physical layer; the data rate is equal to $250 \mathrm{kbps}$.

\section{B. Basic rules}

CoSenS has four basic rules. First, routers have the priority to access the medium over simple nodes. This is achieved using different values of CSMA/CA parameters for each type of node. Second, a router does not transmit packets one by one upon their arrival. Instead, it waits for a period of time, WP, and collects data either from its children or from its neighbor routers. Third, after the end of the WP, the router starts transmitting all packets queued in its buffer in a single burst during the TP. Finally, at the end of the TP, the router restarts another cycle and goes again to the waiting for reception state. WP and TP are illustrated in the example given by the Fig. 1. Full details of these basic rules are given next.

Note that since simple nodes use CSMA/CA, they always transmit data during the WP of their respective fathers because the channel can be free only during that period (CSMA/CA checks the channel status before transmitting).

\section{Waiting Period}

The length of $W P$ is dynamic and depends on the amount of incoming traffic; the higher the traffic volume is, the longer the WP is. In fact, it is continuously adjusted to prevent waste. The WP duration for a router $r$ is given by (1).

$$
W P= \begin{cases}N_{\max } \cdot d_{S}, & \text { if } N_{c}^{r} \neq 0 \\ N_{\max } \cdot d_{R}, & \text { otherwise }\end{cases}
$$

$N_{c}^{r}$ is the number of children associated to the router $r$.

We define, $d_{S}$ and $d_{R}$ as the amount of time needed by a simple node and a router, respectively, to perform collision avoidance procedure $\left(d_{B P}\right)$, sense the channel $\left(t_{C C A}\right)$ and detect that it is Idle (at the first attempt), change the antenna state from receiver to sender $\left(t_{t a t}\right)$, send the packet $\left(d_{p k t}\right)$, wait for an acknowledgment $\left(t_{a c k}\right)$, and receive it $\left(d_{a c k}\right)$ (Fig. 2). $d_{S}$ and $d_{R}$ are calculated by taking the upper bound of $d_{B P}$.

$N_{\max }$ is an integer that has to be estimated using the incoming traffic. Its value gives the duration of the $W P$. It has a minimum value of 1 . The estimation algorithm is described next.

The estimation algorithm: let's define first the set $\Omega \in \mathbb{N}$ as the set of $W P$ s during which the router receives data and $W P_{k}, k \in \Omega$ these $W P$ s. We define also, $U_{k}, k \in \Omega$ as the ratio giving the sum of the service times of received packets $\left(\sum_{i \in E_{k}} \frac{1}{\mu_{i}}, E_{k}\right.$ is the set of received packets during $W P_{k}$ ) divided by $W P_{k}$ duration. $\frac{1}{\mu_{i}}$ is the service time of packet $i$ (Fig. 2). $U_{k}$ is shown in (2). We note that $U_{k}$ can exceed 1 in the case where the router is still receiving a packet from a child or data from a neighbor router while the $W P$ ends (recall that the router is not preemptive).

$$
U_{k}=\frac{\sum_{i \in E_{k}} \frac{1}{\mu_{i}}}{W P_{k}}
$$

Let us denote by $S_{k}$ the exponential moving average of $U_{k}, k \in \Omega$

$$
S_{k}=(1-\alpha) S_{k-1}+\alpha U_{k-1}
$$

$\alpha$ is the smoothing factor. We used a non linear filter where $\alpha$ is greater when $U_{k-1} \geq S_{k-1}$ allowing $S_{k}$ to adapt more swiftly to traffic increase. So, $\alpha$ takes two values; $\alpha_{1}$ if $U_{k-1}<$ $S_{k-1}$ and $\alpha_{2}$ otherwise. In all simulations, the values of $\alpha$ are set to $\alpha_{1}=0.008$ and $\alpha_{2}=0.01$. The study of the values of $\alpha$ is out of the scope of this paper. The computation of $S_{k}$ is similar to the estimation of the round trip time in TCP [8].

$S_{k}$ is evaluated before the beginning of the $k^{\text {th }} W P$ and is used in the Algorithm 1 to determine the value of $N_{\max }$ for the $k^{\text {th }} W P$ (thus its duration). In Algorithm 1,Thr $r_{\max }$ and $T h r_{\min }$ designate the maximum and the minimum threshold of $S_{k}$, respectively. $T h r_{\max }$ and $T h r_{\min }$ allow the increase and the decrease of the WP's duration. In fact, $S_{k}$ calculates the average utilization ratio of the $W P$. If $S_{k}$ exceeds $T h r_{\max }$, the value of the $N_{\max }$ is increased which increases the duration of the WP. if $S_{k}$ decreases below $T h r_{\min }$, the value of $N_{\max }$ is decreased. We can see that changing the $W P$ duration affects the value of $U_{k}$ (2) and so $S_{k}$ which in its turn affects the value of $N_{\max }$. Besides, $S_{k}$ depends also on the traffic load. Hence, our algorithm appears like an iterative process whose objective is to find the good value of $N_{\max }$ that reflects the incoming traffic load while satisfying an utilization ratio between $T h r_{\text {min }}$ and $T h r_{\max }$.

We also define $N M A X$ as the bound of the value of $N_{\max }$ which is used to prevent a high increase in the $W P$ duration. Its value depends on the application and hardware constraints 


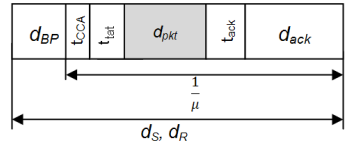

Fig. 2. $d_{S}, d_{R}$ and $\frac{1}{\mu}$ variables.

(e.g., expected transmission interval for nodes, memory size limit for queueing packets).

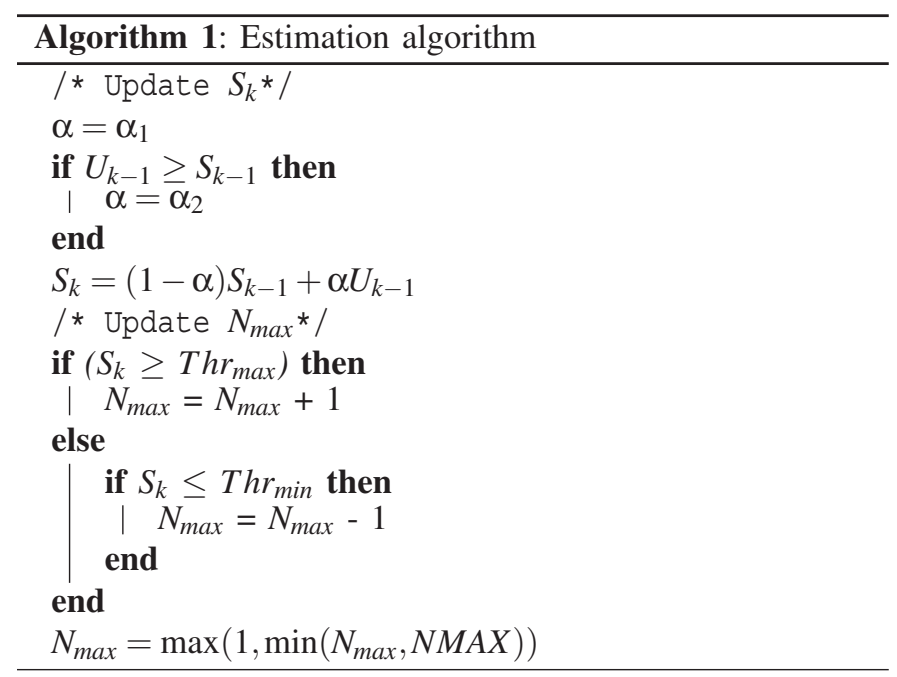

\section{Transmission Period}

The collected data during the waiting period are transmitted in a burst. CSMA/CA protocol is used to transmit only the first packet. Then, the remaining packets are directly transmitted upon the reception of the acknowledgement (ack). We note that the acknowledgments are always enabled in CoSenS. If an ack is not received, the transmitted packet is retransmitted using CSMA/CA protocol again. Burst transmission resumes if the packet is successfully transmitted; The remaining packets will be sent directly after the reception of the ack.

TP has a variable duration which depends on the number of collected packets during WP and the eventual retransmissions. We note that all queued packets are transmitted during the current TP. So, the next WP will start with an empty queue.

\section{PERFormance ANALYSis}

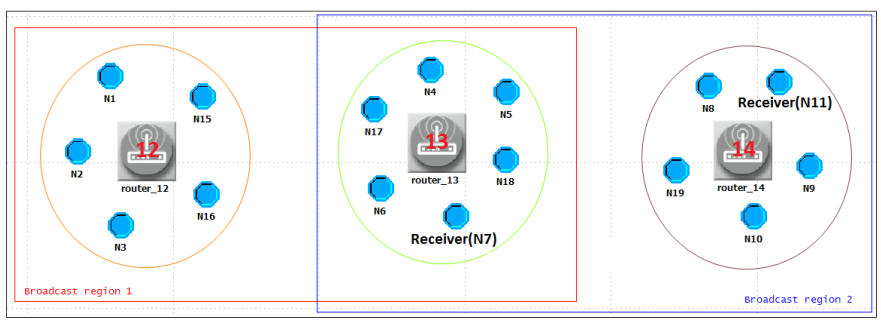

Fig. 3. Line network.
TABLE I

SIMULATION PARAMETERS

\begin{tabular}{|c|c|c|}
\hline \multicolumn{2}{|c|}{ parameter } & value \\
\hline \hline \multirow{4}{*}{ General parameters } & link capacity & $250 \mathrm{kbits} / \mathrm{sec}$ \\
\cline { 2 - 3 } & maximum packet length & $500 \mathrm{bits}$ \\
\cline { 2 - 3 } & packet length & 496 bits (400 bits of data +96 bits of header) \\
\cline { 2 - 3 } & Traffic type & Poisson \\
\cline { 2 - 3 } CoSenS & Simulation duration & $15 \mathrm{~min}$ \\
\hline CSMA/CA & minimum backoff exponent & 2 for routers, 3 for simple nodes \\
\hline \multirow{4}{*}{} & $d_{S}$ & $4.816 \mathrm{~ms}$ \\
\cline { 2 - 3 } & $d_{R}$ & $3.536 \mathrm{~ms}$ \\
\cline { 2 - 3 } & $N M A X$ & 15 \\
\cline { 2 - 3 } & $T h_{\min }$ & 0.28 \\
\cline { 2 - 3 } & $T h_{\max }$ & 0.75 \\
\hline
\end{tabular}

We compare by simulation the performance of CoSenS to IEEE 802.15.4 MAC that uses the unslotted CSMA/CA protocol. Our simulation model is implemented within OPNET[9] (Modeler v14.5). Simulation parameters are given in Table. I, where the values of $d_{S}$ and $d_{R}$ are calculated for the maximum packet length. CSMA/CA parameters are identical for both protocols.

Because of space limitation, only multi-hop line topology is showed. Additional simulations using different topologies, including random ones, can be found in [10]. They give similar results leading to the same conclusion. We consider a network composed of 13 nodes, two receivers and three routers, organized as shown in Fig. 3. Each two neighbor routers hear each other and their respective children. Thus router 13 hears all nodes. Simple nodes are organized in three groups (delimited by circles in the figure). Each one is attached to a router.

All our measurements are plotted as a function of the generated traffic load (named load in the rest of the document) that we define as the traffic generated by all simple nodes application layer. We evaluate the system throughput, end to end delay, successful transmission rate and the selfsynchronization degree between routers which we define as the percentage of the simulated time where their TPs do not overlap.

a) Throughput, end to end delay and successful transmission rate: we consider a first scenario that has two receivers, Receiver(N7) and Receiver(N11). Three simple nodes, each one attached to a router, send data to Receiver(N7) (N3, N6 and N9) while the other ones send data to Receiver(N11).

The results of throughput, end to end delay and successful transmission rate are illustrated in Fig. 4(a), Fig. 4(b) and Fig. 4(c), respectively. CoSenS obtains better throughput than IEEE 802.15.4 MAC. In addition, it saturates at $46 \mathrm{~kb} / \mathrm{s}$ of load compared to a saturation load of $35 \mathrm{~kb} / \mathrm{s}$ for IEEE 802.15.4 MAC. For end-to-end delay, the overall performance of CoSenS is better. As far as the load increases, the difference in terms of end to end delay becomes more and more obvious. For successful transmission rate, the acknowledgment mechanism ensures a $100 \%$ of successful transmissions before the saturation point of both protocols. After that, CoSenS outperforms IEEE 802.15.4 MAC.

b) Organization of WP and TP: we consider a second scenario where all nodes send data to Receiver(N7). This 
TABLE II

SYNCHRONIZATION OF TPS BETWEEN THE THREE ROUTERS

\begin{tabular}{|c||c|c|c|c|c|}
\hline load (kbits/sec) & 8.12 & 18.64 & 27.95 & 34.89 & 46.85 \\
\hline synchronization (\%) & 99.86 & 98 & 91.24 & 76.35 & 26.6 \\
\hline
\end{tabular}

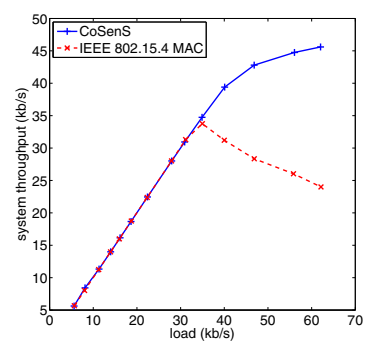

(a) System throughput.
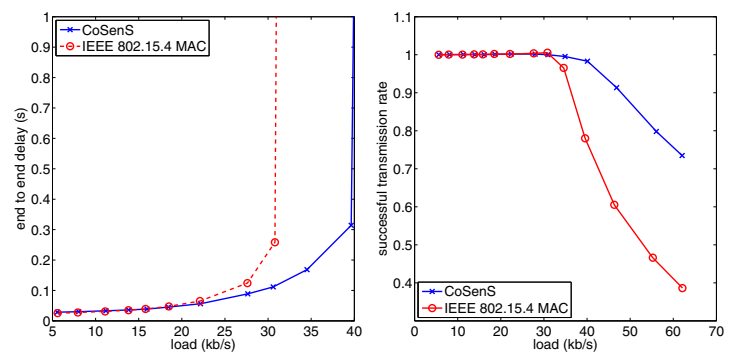

(b) End to end delay.

(c) Successful transmission rate.

Fig. 4. First scenario results.

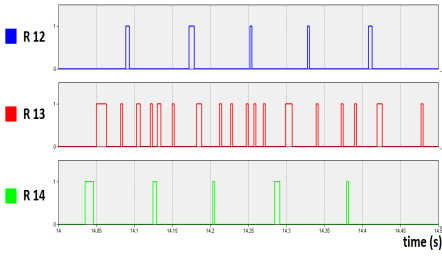

(a) TPs are self-synchronized

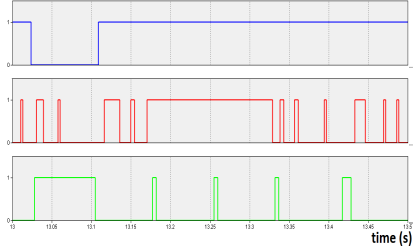

(b) TPs overlap
Fig. 5. Synchronization of the transmission periods. '0' means that the router is in the WP.' 1 ' means that it is in the TP.

scenario suffers from the hidden node problem between routers 12 and 14 since they send data to router 13 . This makes it suitable to study the routers self-synchronization degree because all router TPs need to be synchronized with each other.

Figure 5 shows the organization in time (half a second) of the waiting and transmission periods of the three routers. The TPs are self-synchronized in Fig. 5(a) while they overlap in Fig. 5(b). We measured the self-synchronization degree of the three routers for different workloads. The results are illustrated in Table. II. Under light and normal loads, all routers are well synchronized with each other, avoiding interference. As the load increases, the synchronization percentage decreases. In heavy network load the performance degrades significantly because of transmission collisions. In fact, as described in Sec. II-D, in case of a transmission collision, the router uses
CSMA/CA again to transmit the packet. Since the load is heavy, other routers can gain the access to the medium during the backoff period. The TPs are considered in conflict in this case. Hence, the router must wait until it gains the access to the medium again to resume its TP.

\section{CONCLUSION}

CoSenS is a simple but efficient scheme which improves the performance of the widely used CSMA/CA protocol in wireless communications. Our simulations show that CoSenS greatly enhances throughput, end to end delay and reliability of CSMA/CA. In addition, the proposed scheme inherits the benefits of CSMA/CA, namely its good scalability and lower complexity properties. Since it is implemented as a service, devices that implement CoSenS are compatible with standard compliant ones. Although designed and evaluated for IEEE 802.15.4, the principle of CoSenS could also be directly applicable to other CSMA/CA based protocols (e.g., IEEE 802.11).

The actual estimation algorithm uses several variables that need to be tuned according to the traffic characteristics. The first objective for future work focuses on enhancing this algorithm making it totally self-configuring. Since it queues data before transmitting them, CoSenS enables the support of QoS through the implementation of scheduling and congestion control mechanisms. This is our second objective for future work.

\section{REFERENCES}

[1] Polastre, J., Hill, J., and Culler, D. 2004. Versatile low power media access for wireless sensor networks. In Proceedings of the 2nd international Conference on Embedded Networked Sensor Systems (Baltimore, MD, USA, November 03 - 05, 2004). SenSys '04. ACM, New York, NY, 95107. IEEE/ACM Transactions on , vol.16, no.3, pp.511-524, June 2008.

[2] van Dam, T. and Langendoen, K. 2003. An adaptive energy-efficient MAC protocol for wireless sensor networks. In Proceedings of the 1st international Conference on Embedded Networked Sensor Systems (Los Angeles, California, USA, November 05 - 07, 2003). SenSys '03. ACM, New York, NY, 171-180.

[3] Li, Yanjun and Chen, Chung Shue and Song, Ye-Qiong and Wang, Zhi. Real-time QoS support in wireless sensor networks: a survey. In: 7th IFAC International Conference on Fieldbuses \& Networks in Industrial \& Embedded Systems - FeT'2007, Toulouse France, 2007.

[4] IEEE-TG15.4 (2006). Part 15.4: Wireless Medium Access Control (MAC) and Physical Layer (PHY) specifications for low-rate Wireless Personal Area Networks (LR-WPANs). IEEE standard for information technology.

[5] ZigBee Specification Document 053474r17.http://www.zigbee.org

[6] Koubaa, A., Cunha, A., and Alves, M. 2007. A Time Division Beacon Scheduling Mechanism for IEEE 802.15.4/Zigbee Cluster-Tree Wireless Sensor Networks. In Proceedings of the 19th Euromicro Conference on Real-Time Systems (July 04 - 06, 2007). ECRTS. Washington, DC.

[7] WirelessHart. http://www.hartcomm.org/.

[8] Jon Postel, Transport Control Protocol, RFC 793, September 1981

[9] OPNET, "OPNET Simulator, v 14.5, http://www.opnet.com."

[10] B. Nefzi, Y.-Q. Song. "CoSenS reference guide". INRIA Technical report. December 2009 http://hal.archives-ouvertes.fr/inria-00443594/en/.

[11] IEEE 802.11b/D3.0, Wireless LAN Medium Access Control (MAC) and Physical (PHY) Layer Specification: High Speed Physical Layer Extensions in the $2.4 \mathrm{GHz}$ Band, 1999.

[12] Nefzi, B.; Cruz-Sanchez, H.; Ye-Qiong Song; , "SCSP: An energy efficient network-MAC cross-layer design for wireless sensor networks," Local Computer Networks, 2009. LCN 2009. IEEE 34th Conference on , vol., no., pp.1061-1068, 20-23 Oct. 2009. 